\title{
1 EINLEITUNG UND ZIELSETZUNGEN
}

Im Anschluss an das Projekt „ISWI-MAB - Integrated Sustainable Wildlife Management in the Biosphere Reserve Wienerwald" (Reimoser et al. 2008), in dem Prinzipien, Kriterien und Indikatoren für den nachhaltigen Umgang mit Wildtieren und deren Lebensräumen in Bezug auf die Landnutzungssektoren Jagd, Land- und Forstwirtschaft sowie Freizeit-und Erholungsmanagement entwickelt wurden, fokussierte das vorliegende Projekt „IESP -Towards Integrated Ecological Spatial Planning for the Wienerwald Biosphere Reserve" insbesondere auf die Ermittlung, Analyse und räumliche Zuordnung der Konfliktpotenziale zwischen Wildtieren und Freizeitaktivitäten sowie die systematische Ableitung ortsbezogener Maßnahmen für das Konfliktmanagement.

\section{Thematischer Fokus}

Das zentrale Thema des Projekts IESP sind Konfliktpotenziale und Konflikte zwischen den Lebensraumansprüchen von Wildtieren und menschlichen Landnutzungsansprüchen. Mit dem Fokus auf naturraumbasierten Freizeit- und Erholungsaktivitäten sollten am Beispiel der Biosphärenparkregion Wienerwald die komplexen Interaktionsfelder zwischen Freizeitnutzungen einerseits und Wildtierarten andererseits untersucht, Konfliktpotenziale und deren Auswirkungen in ihren räumlichen und zeitlichen Dimensionen erfasst und analysiert sowie Handlungsstrategien und -optionen für ein räumlich orientiertes Konfliktmanagement erarbeitet werden. Der thematische Fokus und der konkrete Untersuchungsraum des Biosphärenparks Wienerwald stehen dabei exemplarisch und stellvertretend für Mensch-WildtierKonflikte in Mehrfachnutzungs-Kulturlandschaften.

\section{Projektziele}

Das Hauptziel des Projekts war die Entwicklung und Bereitstellung von fachlichen und methodischen Grundlagen für ein integratives und vorausschauendes Management von Konflikten zwischen den Lebensraumansprüchen von Wildtieren und Landnutzungsansprüchen des Menschen am Beispiel von naturraumbasierten Freizeitnutzungen im Biosphärenpark Wienerwald und unter Anwendung räumlich bzw. raumplanerisch orientierter Ansätze. Das übergeordnete Ziel ist die Verbesserung der Grundlagen für eine integrative, nachhaltige Landnutzung, das heißt insbesondere für eine systematische Abstimmung unterschiedlicher Landnutzungsaktivitäten im Hinblick auf nachhaltige Ressourcennutzung, Erhaltung der Biodiversität und präventive Konfliktminimierung.

Das Hauptziel lässt sich in folgende konkrete Teilziele gliedern:

- Die Erfassung, Charakterisierung und Kartierung der Verteilung und Lebensraumnutzung von Indikator-Wildtierarten (in Intensivuntersuchungsgebieten und auf biosphärenparkweiter Maßstabsebene).

- Die Erfassung, Modellierung, Charakterisierung und Kartierung von Raumansprüchen und Nutzungsintensitäten bzw. -wahrscheinlichkeiten von Indikator-Freizeitaktivitäten (in Intensivuntersuchungsgebieten und auf biosphärenparkweiter Maßstabsebene).

- Die Ermittlung, Modellierung, Analyse, Typisierung und Kartierung von räumlichen Konfliktpotenzialen zwischen Indikatorarten und Indikatoraktivitäten (in Intensivuntersuchungsgebieten und auf biosphärenparkweiter Maßstabsebene).

- Die Entwicklung und Bereitstellung von Konzepten, Analyse- und Kartierungsmethoden sowie entscheidungsunterstützenden Werkzeugen als Grundlage für ein raumplanerisch basiertes Konfliktmanagement. 
- Die Entwicklung von Handlungsstrategien, Maßnahmenoptionen und Entscheidungshilfen (,Maßnahmenbaukasten“) für ein räumlich orientiertes, integratives Konfliktmanagement:

o Katalog von Managementoptionen und Empfehlungen für das Besuchermanagement, für Information, Kommunikation und Öffentlichkeitsarbeit, für sektorale Landnutzungsbereiche sowie für Monitoring und adaptives Management.

o Empfehlungen zur Priorisierung, Auswahl und räumlich differenzierten Zuordnung von Managementzielen und -maßnahmen zu unterschiedlichen Konfliktpotenzialausprägungen.

- Empfehlungen für einen umsetzungsorientierten Folgeprozess zur Einrichtung eines raumplanerisch orientierten Konfliktmanagements im Biosphärenpark Wienerwald.

\section{Methodische Ausrichtung}

Dem Projekt liegt eine Reihe von grundsätzlichen methodischen Ansätzen und konzeptiven Überlegungen zu Grunde, deren einleitende Erwähnung für das Verständnis des vorliegenden Berichts hilfreich erscheint:

- Partizipativer Ansatz und Stakeholder-Dialog: Basierend auf der Einbettung in ein partizipatives Forschungsdesign wurden die Projektarbeiten in enger Zusammenarbeit mit regionalen Stakeholdern durchgeführt. Hierbei wurden insbesondere die Beteiligungsform des Stakeholderdialogs sowie konsultative Methoden eingesetzt.

- Indikatorenbasierter Ansatz: Die Erfassung und Analyse von Konfliktpotenzialen und das diesbezügliche Konzept für das Konfliktmanagement stützen sich auf IndikatorWildtierarten und Indikator-Freizeitaktivitäten. Die ausgewählten Indikatorarten und Indikatoraktivitäten erfüllen Schirmfunktionen für ein breites Spektrum weiterer Tierarten und menschlicher Aktivitäten im Naturraum; ihre Verknüpfung steht stellvertretend für Interaktionen zwischen Wildtieren und Landnutzungsansprüchen. Ein wesentliches Auswahlkriterium an die Indikatorarten bildete ihre grundsätzliche Störungssensibilität gegenüber Freizeitaktivitäten, während für die Auswahl der Indikatoraktivitäten vorrangig ein relevanter Störungseinfluss auf Wildtierarten maßgeblich war. Die räumliche und zeitliche Überlagerung der Verteilung und Lebensraumnutzung der Indikatorarten einerseits mit den Nutzungsmustern und und Nutzungsintensitäten der Indikatoraktivitäten andererseits indiziert im Projekt das Vorhandensein von Konfliktpotenzialen.

- Räumliche Untersuchungsebenen: Die Untersuchungen wurden im Projekt auf zwei vorrangigen Maßstabsebenen durchgeführt: auf örtlicher Ebene in Intensivuntersuchungsgebieten und auf Ebene der gesamten Biosphärenparkregion. Das anhand der Intensivuntersuchungsgebiete gewonnene Wissen über Konfliktstrukturen und mechanismen bildete $u$. a. eine wesentliche Grundlage für die Methodenentwicklung zur biosphärenparkweiten Konfliktpotenzialmodellierung und zur Entwicklung von Maßnahmenoptionen für das Management.

- GIS-gestützte Modellierungen, Analysen und Kartendarstellungen: Bei der Darstellung der räumlichen Verteilungsmuster von Indikatorarten und -aktivitäten und bei der Ermittlung und Analyse von Konfliktpotenzialen wurde stark mit GIS-analytischen Methoden gearbeitet. Da konkrete Daten zu Freizeitaktivitäten für den gesamten Biosphärenpark weitgehend fehlen, wurden Freizeitnutzerströme in der Untersuchungsregion auf Basis vorhandener empirischer Informationen und theoretischer, literaturgestützter Annahmen im Geographischen Informationssystem modelliert. Die hierfür entwickelte Methodik erlaubt es, den räumlichen Bezug von Indikatorarten, Indikatoraktivitäten und Konfliktpotenzialen in GIS-basierten Kartendarstellungen zu visualisieren. 
- Berücksichtigung funktionaler Zusammenhänge zwischen Biosphärenpark und Umland: Ausgewählte funktionelle Zusammenhänge des Biosphärenparks Wienerwald mit der Umgebungsregion werden gezielt berücksichtigt. Dies erfolgt einerseits über großräumige ökologische Konnektivitätsaspekte bei relevanten Indikatorarten (z. B. großräumige Mobilitätserfordernisse des Rotwilds im Alpen-Karpaten-Kontext; bestehende Kernhabitate des Auerhuhns außerhalb der Biosphärenparkgrenzen als potenzielle Quellpopulationen für eine Wiederansiedlung innerhalb des Biosphärenparks). Andererseits wird die Bevölkerungs- und Siedlungsentwicklung in der Großregion als wesentlicher Quellfaktor für die (zukünftige) Erholungsnachfrage im Biosphärenpark einbezogen und auch als Ursache von Lebensraumeinengung und Habitatfragmentierung thematisiert.

- Berücksichtigung weiterer Landnutzungen: Neben Freizeitaktivitäten werden die Einflüsse anderer Landnutzungen auf Wildtiere, Lebensräume und durch Freizeitbetrieb induzierte Konfliktpotenziale im Projekt indirekt berücksichtigt. Raumplanung, Siedlungsentwicklung und Infrastrukturplanung steuern maßgeblich die Faktoren (der Quellgebiete) der Erholungsnachfrage sowie die Zugänglichkeit der Landschaft für Freizeitnutzer. Gemeinsam mit Land- und Forstwirtschaft prägen sie zudem die Landschaftsstruktur und -ausstattung, und damit sowohl die Erholungseignung der Landschaft als auch die Habitatbedingungen für Wildtiere. Die Jagdwirtschaft wiederum wird aufgrund ihres direkten Einflusses auf Wildpopulationen und -verhalten als integraler Faktor der dem Jagdrecht unterliegenden Indikatorarten (Rotwild, Schwarzwild, Auerhuhn) behandelt. Alle genannten Landnutzungsbereiche, einschließlich des Naturschutzes, werden aufgrund ihres Einflusses auf Konfliktpotenziale und Konfliktmanagement daher auch in eigenen Managementempfehlungen adressiert.

\section{Adressaten der Ergebnisse}

Die Ergebnisse des Projekts richten sich an alle Akteure im Biosphärenpark, die an der Entstehung von Konfliktpotenzialen beteiligt sind, von Konfliktfolgen betroffen sind oder Einflussmöglichkeit auf Konfliktmanagementprozesse besitzen. Zielgruppen umfassen daher beispielsweise das Biosphärenpark Wienerwald Management, zuständige Entscheidungsträger in Landes- und Bezirksverwaltungen, Gemeindevertretungen, Vertreter der unterschiedlichen Freizeitnutzergruppen, Grundeigentümer und -bewirtschafter, Vereine und Interessenvertretungen, etc. Hinsichtlich der sektoralen bzw. fachlichen Zuständigkeiten werden insbesondere die Bereiche Freizeitwirtschaft, Natur- und Artenschutz, Raumplanung, Forstwirtschaft, Landwirtschaft und Jagdwirtschaft adressiert. 